\title{
Successful Treatment of a Patient with anti-PD1 Antibody-Resistant Advanced Mucosal Melanoma with Nivolumab, Ipilimumab plus Denosumab Combination Therapy
}

\author{
Taku Fujimura Yumi Kambayashi Kentaro Ohuchi Ryo Amagai \\ Yota Sato Kayo Tanita Akira Hashimoto Setsuya Aiba \\ Department of Dermatology, Tohoku University Graduate School of Medicine, \\ Sendai, Japan
}

\author{
Keywords \\ Mucosal melanoma - Bone metastasis - Nivolumab plus ipilimumab combination therapy · \\ Denosumab
}

\section{Abstract}

Since the incidence of mucosal melanoma is higher in the Japanese population compared to Caucasians, and since mucosal melanoma possesses a lower mutation burden compared to cutaneous melanoma, the efficacy of anti-PD1 antibody (Ab) monotherapy for mucosal melanoma is limited. Therefore, other targeting molecules that enhance the anti-tumor effects of immune checkpoint inhibitors are needed. In this report, we present a case with anti-PD1 Abresistant recurrent malignant melanoma of the nasal cavity successfully treated with nivolumab, ipilimumab plus denosumab combination therapy.

\section{Introduction}

Since the incidence of mucosal melanoma is higher in the Japanese population compared to Caucasians [1], and since mucosal melanoma possesses a lower mutation burden compared to cutaneous melanoma [2], the efficacy of anti-PD1 antibody (Ab) monotherapy for mucosal melanoma is limited $[3,4]$. In addition, the efficacy of ipilimumab monotherapy in patients with nivolumab-resistant melanoma is extremely low after objective tumor progression [5]. 


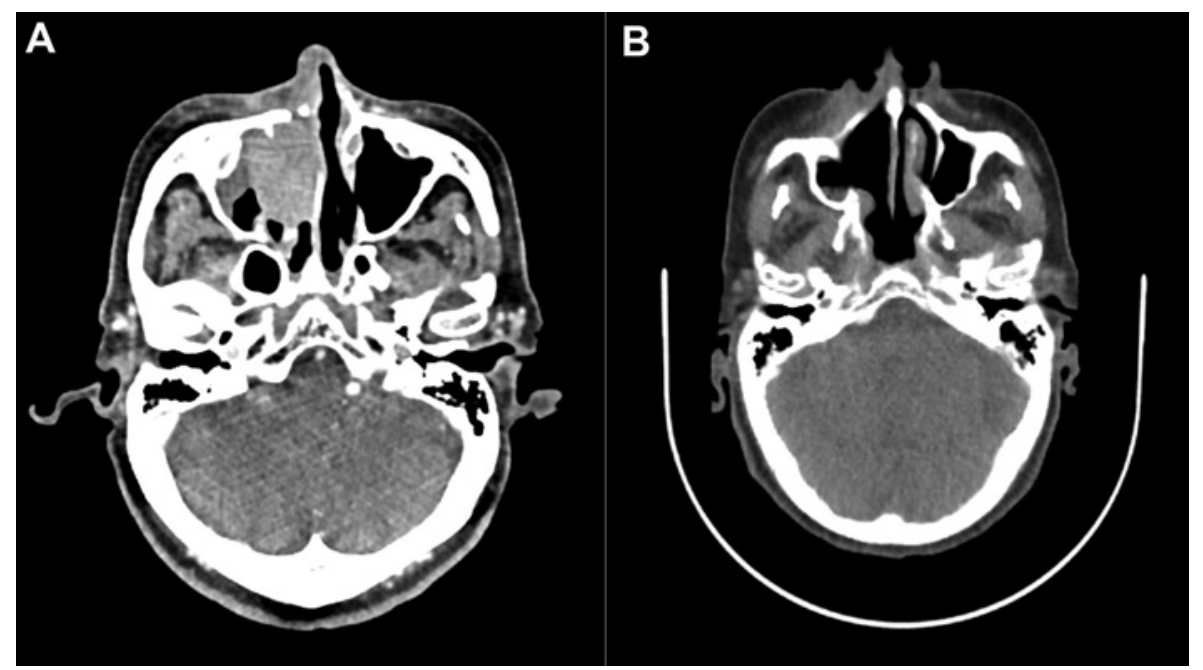

Fig. 1. CT scan before radiotherapy: local recurrence of melanoma, $36.80 \times 26.78 \mathrm{~mm}$ in size, in the nasal cavity (A). MRI at 2 months after IMRT treatment: regression of the tumor (B).

Therefore, other targeting molecules that enhance the anti-tumor effects of immune checkpoint inhibitors (ICIs) are needed. In this report, we present a case with anti-PD1 Ab-resistant recurrent malignant melanoma of the nasal cavity successfully treated with nivolumab, ipilimumab plus denosumab combination therapy.

\section{Case Report}

The patient was an 81-year-old Japanese woman described in the Journal of Dermatology in 2019 [6]. Twenty-one months after the administration of pembrolizumab monotherapy, follow-up computed tomography (CT) scan revealed the local recurrence of melanoma, 36.80 $\times 26.78 \mathrm{~mm}$ in size, in the nasal cavity (Fig. 1A). Since the recurrent tumor was limited to the nasal cavity, we employed intensity-modulated radiotherapy (IMRT) using CyberKnife with 45 Gy in 9 fractions. Two months after the radiotherapy, magnetic resonance imaging (MRI) revealed regression of the tumor (Fig. 1B). However, 3 months after tumor regression, followup positron emission tomography (PET)-CT revealed multiple metastases in the lungs, scapula, and subcutaneous lesions (Fig. 2A). Since the melanoma was BRAFV600E mutation negative, nivolumab ( $80 \mathrm{mg} / \mathrm{kg} /$ every 3 weeks) was given in combination with ipilimumab ( $3 \mathrm{mg} / \mathrm{kg} /$ every 3 weeks) for 4 cycles without any adverse events. In addition, since this patient showed metastatic melanoma of the bone, we administered denosumab $120 \mathrm{mg}$ every month. Three months after the first administration of nivolumab plus ipilimumab combination therapy, the multiple metastases in the lungs, scapula, and subcutaneous lesions had regressed (Fig. 2B). We continued to administer pembrolizumab ( $240 \mathrm{mg} / \mathrm{kg} /$ every 3 weeks), and there was no evidence of recurrence 6 months after achieving complete remission.

\section{Discussion}

The combination or sequential administration of nivolumab and ipilimumab with a planned switch is among the most effective chemotherapies against advanced melanoma [7, 8], but the efficacy of ipilimumab monotherapy in patients with nivolumab-resistant cuta- 
Fujimura et al.: Advanced Mucosal Melanoma and Nivolumab, Ipilimumab plus Denosumab Combination Therapy
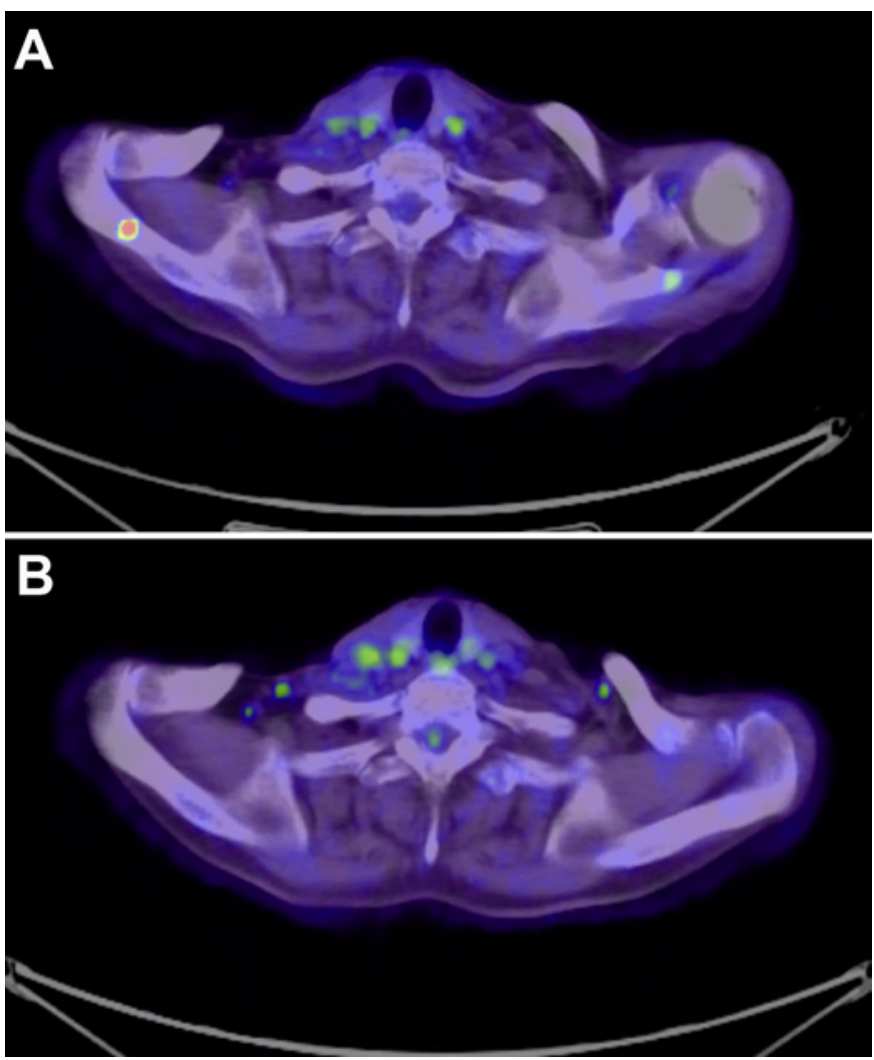

Fig. 2. PET-CT image: metastasis at the scapula before (A) and after (B) combination therapy.

neous and mucosal melanoma is low after objective tumor progression compared to its efficacy in patients with planned-switched treatment $[5,9]$. These reports suggested that the efficacy of nivolumab plus ipilimumab combination therapy in anti-PD1 Ab therapy-resistant patients is lower than that in anti-PD1 Ab therapy-naïve patients. In addition, recently, Hamid et al. [4] has reported the results of a case series with mucosal melanoma treated with pembrolizumab monotherapy. The objective response rate to pembrolizumab for ipilimumab therapy-naïve mucosal melanoma patients was $22 \%$, suggesting a poor prognosis for mucosal melanoma compared to cutaneous melanoma patients [10]. Therefore, additional methods to enhance the anti-tumor effects of ICIs in patients with mucosal melanomas are needed.

To enhance the anti-tumor effects of anti-PD1 Abs, not only the induction of CD8+ T cells in the tumor lesion $[11,12]$, but also other targeting molecules that enhance the anti-tumor effects of ICIs should be taken into account [13]. Recently, Ahern et al. [14,15] have highlighted the therapeutic effects of co-administration of anti-RANKL Abs with ICIs, such as anti-PD1 Abs and anti-CTLA4 Abs, against melanoma by the suppression of RANKL ${ }^{+} \mathrm{PD} 1^{\text {high }} \mathrm{CD} 8 \mathrm{~T}$ cells in a B16F10 mouse melanoma model. They concluded that anti-RANKL Abs could enhance the anti-melanoma effects of ICIs. Indeed, in clinics, anti-RANKL Abs enhanced the therapeutic effects of ipilimumab in patients with terminal-stage metastatic melanoma [16, 17]. These reports suggested that denosumab might improve the therapeutic effects of nivolumab plus ipilimumab combination therapy against advanced anti-PD1 Ab-resistant mucosal melanoma.

In this report, we described a case of anti-PD1 Ab-resistant advanced mucosal melanoma treated with nivolumab, ipilimumab plus denosumab combination therapy. Our present case suggested that nivolumab, ipilimumab plus denosumab combination therapy is not only useful for conventional cutaneous melanoma as we previously reported [17], but also useful for recurrent anti-PD1 Ab-resistant mucosal melanoma as a second-line therapy. 
Fujimura et al.: Advanced Mucosal Melanoma and Nivolumab, Ipilimumab plus Denosumab Combination Therapy

\section{Statement of Ethics}

The patient gave written informed consent.

\section{Disclosure Statement}

The authors have no conflicting interests to declare.

\section{Funding Sources}

This study was supported in part by the Japan Agency for Medical Research and Development $(19 \mathrm{~cm} 0106434 \mathrm{~h} 0002)$.

\section{Author Contributions}

Taku Fujimura designed the research study. Taku Fujimura, Yumi Kambayashi, Ohuchi Kentaro, Ryo Amagai, Sato Yota, Tanita Kayo, and Akira Hashimoto treated the patient and acquired the clinical data. Taku Fujimura wrote the manuscript. Taku Fujimura and Setsuya Aiba supervised the study.

\section{References}

1 Fujisawa Y, Yoshikawa S, Minagawa A, Takenouchi T, Yokota K, Uchi H, et al. Clinical and histopathological characteristics and survival analysis of 4594 Japanese patients with melanoma. Cancer Med. 2019;8:2146-56.

2 Hayward NK, Wilmott JS, Waddell N, Johansson PA, Field MA, Nones K, et al. Whole-genome landscapes of major melanoma subtypes. Nature. 2017;545:175-80.

3 D'Angelo SP, Larkin J, Sosman JA, Lebbé C, Brady B, Neyns B, et al. Efficacy and safety of nivolumab alone or in combination with ipilimumab in patients with mucosal melanoma: a pooled analysis.J Clin Oncol. 2017;35:22635.

4 Hamid O, Robert C, Ribas A, Hodi FS, Walpole E, Daud A, et al. Antitumour activity of pembrolizumab in advanced mucosal melanoma: a post-hoc analysis of KEYNOTE-001, 002, 006. Br J Cancer. 2018;119:670-4.

5 Fujisawa Y, Yoshino K, Otsuka A, Funakoshi T, Uchi H, Fujimura T, et al. Retrospective study of advanced melanoma patients treated with ipilimumab after nivolumab: analysis of 60 Japanese patients. J Dermatol Sci. 2018;89:60-6.

6 Fujimura T, Kambayashi Y, Sato Y, Tanita K, Tono H, Lyu C, et al. Successful treatment of unresectable recurrent programmed death ligand 1 moderately-expressing malignant melanoma of the nasal cavity with pembrolizumab monotherapy. J Dermatol. 2019;46:e260.

7 Larkin J, Chiarion-Sileni V, Gonzalez R, Grob JJ, Cowey CL, Lao CD, et al. Combined nivolumab and ipilimumab or monotherapy in untreated melanoma. N Engl J Med. 2015;373:23-34.

8 Weber JS, Gibney G, Sullivan RJ, Sosman JA, Slingluff CL Jr, Lawrence DP, et al. Sequential administration of nivolumab and ipilimumab with a planned switch in patients with advanced melanoma (CheckMate 064): an open-label, randomised, phase 2 trial. Lancet Oncol. 2016;17:943-55.

9 Saijo K, Imai H, Ouchi K, Okada Y, Sato Y, Komine K, et al. Therapeutic benefits of ipilimumab among Japanese patients with nivolumab-refractory mucosal melanoma: a case series study. Tohoku J Exp Med. 2019;248:3743.

10 Carlino MS, Long GV, Schadendorf D, Robert C, Ribas A, Richtig E, et al. Outcomes by line of therapy and programmed death ligand 1 expression in patients with advanced melanoma treated with pembrolizumab or ipilimumab in KEYNOTE-006: a randomised clinical trial. Eur J Cancer. 2018;101:236-43.

11 Fujimura T, Okuyama R, Ohtani T, Ito Y, Haga T, Hashimoto A, et al. Perilesional treatment of metastatic melanoma with interferon-beta. Clin Exp Dermatol. 2009;34:793-9.

12 Kakizaki A, Fujimura T, Furudate S, Kambayashi Y, Yamauchi T, Yagita H, et al. Immunomodulatory effect of peritumorally administered interferon-beta on melanoma through tumor-associated macrophages. Oncoimmunology. 2015;4:e1047584. 
13 van Dam PA, Verhoeven Y, Trinh XB, Wouters A, Lardon F, Prenen H, et al. RANK/RANKL signaling inhibition may improve the effectiveness of checkpoint blockade in cancer treatment. Crit Rev Oncol Hematol. 2019;133:85-91.

14 Ahern E, Harjunpää H, O’Donnell JS, Allen S, Dougall WC, Teng MWL, et al. RANKL blockade improves efficacy of PD1-PD-L1 blockade or dual PD1-PD-L1 and CTLA4 blockade in mouse models of cancer. Oncoimmunology. 2018;7:e1431088.

15 Ahern E, Harjunpää H, Barkauskas D, Allen S, Takeda K, Yagita H, et al. Co-administration of RANKL and CTLA4 antibodies enhances lymphocyte-mediated antitumor immunity in mice. Clin Cancer Res. 2017;23:5789-801.

16 Smyth MJ, Yagita H, McArthur GA. Combination anti-CTLA-4 and anti-RANKL in metastatic melanoma. J Clin Oncol. 2016;34:e104-6.

17 Yoshida S, Fujimura T, Kambayashi Y, Amagai M, Hashimoto A, Aiba S. Successful treatment of multiple metastatic melanoma with nivolumab, ipilimumab plus denosumab combined therapy. Case Rep Oncol. 2019; 12:829-33. 